\title{
Study of strong turbulence effects for optical wireless links
}

Heba Yuksel

Hasim Meric

Fulya Kunter 


\title{
Study of Strong Turbulence Effects for Optical Wireless Links
}

\author{
HEBA YUKSEL ${ }^{1}$, HASIM MERIC ${ }^{2}$, FULYA KUNTER ${ }^{3}$ \\ ${ }^{1}$ Bogazici University, Dept. of Electrical-Electronics Eng. Istanbul, Turkey \\ e-mail: heba.yuksel@boun.edu.tr \\ ${ }^{2}$ Bilkent University, Dept. of Electrical-Electronics Eng. Ankara, Turkey \\ e-mail: hasim@bilkent.edu.tr \\ ${ }^{3}$ Marmara University, Dept. of Electrical-Electronics Eng. Istanbul, Turkey \\ e-mail: fulya.kunter@marmara.edu.tr
}

\begin{abstract}
Strong turbulence measurements that are taken using real time optical wireless experimental setups are valuable when studying the effects of turbulence regimes on a propagating optical beam. In any kind of FSO system, for us to know the strength of the turbulence thus the refractive index structure constant, is beneficial for having an optimum bandwidth of communication. Even if the FSO Link is placed very well-high-above the ground just to have weak enough turbulence effects, there can be severe atmospheric conditions that can change the turbulence regime. Having a successful theory that will cover all regimes will give us the chance of directly processing the image in existing or using an additional hardware thus deciding on the optimum bandwidth of the communication line at firsthand.

For this purpose, Strong Turbulence data has been collected using an outdoor optical wireless setup placed about 85 centimeters above the ground with an acceptable declination and a path length of about 250 meters inducing strong turbulence to the propagating beam. Variations of turbulence strength estimation methods as well as frame image analysis techniques are then been applied to the experimental data in order to study the effects of different parameters on the result. Such strong turbulence data is compared with existing weak and intermediate turbulence data. Aperture Averaging Factor for different turbulence regimes is also investigated.
\end{abstract}

Keywords: Free Space Optical Communication, Optical Wireless Link, Strong Turbulence, refractive index structure constant $\mathrm{C}_{\mathrm{n}}{ }^{2}$, Aperture Averaging, intensity variance.

\section{INTRODUCTION}

Studying the effects of turbulence on a propagating beam on a horizontal direction is essential for every system that have the substance of air propagation. In any kind of FSO system, strength of the turbulence thus the refractive index structure constant, is commanding on our bandwidth of communication [1].

Even in commercial FSO Links that are placed very well-high-above the ground just to have weak enough turbulence effects, there can be severe atmospheric conditions that can increase the turbulence strength or a light rain that will smooth out the spatial effects of optical scintillation within the propagation of the beam path. The weather conditions are mainly effective on the strength of the turbulence [2,3]. Strong turbulence theory is applied only when the intensity variance is bigger than ' 1 ' thus the intensity variance has to be measured beforehand $[4,5]$.

Having a successful theory that will cover all regimes in terms of turbulence strength will give us an upper hand on optimizing the communication system. Weak turbulence theory is well studied and backed up with the experimental data. In the preceding sections, data acquisition techniques for moderate-to-strong turbulence regimes and Aperture Averaging factor results for various turbulence strengths will be given.

Laser Communication and Propagation through the Atmosphere and Oceans, edited by Alexander M. J. van Eijk, Christopher C. Davis, Stephen M. Hammel, Arun K. Majumdar, Proc. of SPIE Vol. 8517, 85170I - (c) 2012 SPIE · CCC code: 0277-786X/12/\$18 - doi: 10.1117/12.929604 
Scintillation is an inevitable phenomenon that occurs on any optical propagation system with a considerable distance. Tiny bit of temperature change along the optical path is all that is needed for fluctuations in the refractive index constant in the air. Intensity variations can be expressed as,

$$
\sigma_{I}^{2}=\frac{\left\langle I^{2}\right\rangle-\langle I\rangle^{2}}{\langle I\rangle^{2}}
$$

where $I$ is the Intensity at the receiving point detector.

The Rytov method provides a solution for the variance of the log intensity fluctuations seen by a point detector for a plane wave in weak turbulence conditions,

$$
\sigma_{\ln I_{R}}^{2}=\left\langle(\ln I-\langle\ln I\rangle)^{2}\right\rangle=1.23 C_{n}^{2} k^{7 / 6} L^{11 / 6}
$$

Although the Rytov variance stands for weak turbulence fluctuations, in the literature it is considered a measure of turbulence strength by either increasing the refraction structure parameter $\left(\mathrm{C}_{\mathrm{n}}{ }^{2}\right)$ or the propagation path length $(\mathrm{L})$ [6]. Behavior of the scintillation on the receiver point is somewhat interesting. Experimental and theoretical results showed that scintillation at the receiver increases with the Rytov variance until a maximum value. Then the weak nature of the turbulence decreases and strong turbulence inhomogeneities start to have a larger effect on the scintillation so by multiple scattering the scintillation slowly decreases and reaches to a saturation level [6].

In weak turbulence theory, it has been assumed that the correlation length of the intensity fluctuations is on the order of the first fresnel zone, $\sqrt{\frac{L}{k}}$, which is $9.8 \mathrm{~mm}$ for our weak turbulent experiments where the Length of the path is 951 meters and $\lambda=\approx 633 \mathrm{~nm}$ and $\sqrt{\frac{L}{k}}$ is $3.5 \mathrm{~mm}$ for our 120 meters link at Bilkent University with the same $\lambda$. However in strong turbulence regimes the coherent beam becomes increasingly less coherent as it propagates and scintillation appears like the outcome of multiple sources with distinct but random phases [7].

Andrews and Phillips developed a model for the plane wave case that is valid under all fluctuation conditions. This was done through replacing the Kolmogorov spectrum with the effective Kolmogorov spectrum [ 8, 9,10],

$$
\begin{aligned}
\Phi_{n, e}(\kappa) & =\Phi_{n}(\kappa)\left[G_{x}(\kappa)+G_{y}(\kappa)\right\rfloor \\
& =0.033 C_{n}^{2} \kappa^{-11 / 3}\left[G_{x}(\kappa)+G_{y}(\kappa)\right],
\end{aligned}
$$

where the large-scale filter function that passes only spatial frequencies $\kappa<\kappa_{x}$ is,

$$
G_{x}(\kappa)=\exp \left(-\frac{\kappa^{2}}{\kappa_{x}^{2}}\right)
$$

and the small-scale filter function passing only $\kappa>\kappa_{y}$ is,

$$
G_{y}(\kappa)=\frac{\kappa^{11 / 3}}{\left(\kappa^{2}+\kappa_{y}^{2}\right)^{11 / 6}}
$$

Using the modified Rytov theory, the scintillation index is defined in terms of the large-scale $\sigma_{\ln x}^{2}$ and small scale $\sigma_{\ln y}^{2} \log$ irradiance fluctuations which are described in detail in Ref. [8], 


$$
\sigma_{I}^{2}=\exp \left(\sigma_{\ln x}^{2}+\sigma_{\ln y}^{2}\right)-1
$$

The scintillation index for a plane wave, excluding inner scale effects, is then defined to be [8,9],

$$
\sigma_{I}^{2}=\exp \left[\frac{0.49 \sigma_{R}^{2}}{\left(1+1.11 \sigma_{R}^{12 / 5}\right)^{7 / 6}}+\frac{0.51 \sigma_{R}^{2}}{\left(1+0.69 \sigma_{R}^{12 / 5}\right)^{5 / 6}}\right]-1
$$

where $\sigma_{R}^{2}$ is the Rytov variance for a plane wave. Equation (7) reduces to the Rytov variance under weak turbulence conditions, and to the Andrews asymptotic model under strong turbulence conditions.

For a spherical wave, the scintillation index for zero inner scale effects is defined by $[8,9]$,

$$
\sigma_{I}^{2}=\exp \left[\frac{0.20 \sigma_{R}^{2}}{\left(1+0.19 \sigma_{R}^{12 / 5}\right)^{7 / 6}}+\frac{0.20 \sigma_{R}^{2}}{\left(1+0.23 \sigma_{R}^{12 / 5}\right)^{5 / 6}}\right]-1 .
$$

The main assumption is that the small-scale fluctuations are modulated by large-scale fluctuations of the wave. So the theory above treats the atmosphere like a linear spatial filter which causes the loss of transverse spatial coherence of the propagating wave by getting rid of scale sizes that are defined by the coherence radius and the scattering disk.

The heuristic approach based on the trusted physical arguments presented by Andrews et al. for Aperture Averaging Factor for moderate to strong turbulent regimes is given below.

$$
\sigma_{I n_{-} x, p l}^{2}\left(D, l_{0}\right) \cong 0.16 \sigma_{1}^{2}\left(\frac{\hat{\eta_{x}} Q_{1}}{\hat{\eta}_{x}+Q_{1}}\right)^{7 / 6}\left[1+1.753\left(\frac{\hat{\eta_{x}}}{\hat{\eta}_{x}+Q_{1}}\right)^{1 / 2}-0.252\left(\frac{\hat{\eta_{x}}}{\hat{\eta}_{x}+Q_{1}}\right)^{7 / 12}\right] \text { (9) }
$$

where, $Q_{1}=l \kappa_{l}^{2} / k=10.89 / k l_{0}^{2}, d=\sqrt{\frac{k D^{2}}{4 L}}$ and

$$
\sigma_{1}^{2}=1.23 C_{n}^{2} k^{7 / 6} L^{11 / 6}
$$

being the Rytov variance and,

$$
\begin{aligned}
& \eta_{x}=\frac{2.61}{1+0.45 \sigma_{1}^{2} Q_{l}^{1 / 6}} \\
& \hat{\eta}_{x}=\frac{\eta_{x}}{1+d^{2} \eta_{x} / 4}=\frac{2.61}{1+0.623 d^{2}+0.45 \sigma_{I}^{2} Q_{1}^{1 / 6}} .
\end{aligned}
$$

So overall, the scintillation index parameter for moderate to strong turbulent regime with plane wave approximation is given,

$$
\sigma_{I, p l}^{2}\left(D, l_{0}\right)=\exp \left[\sigma_{I n_{-} x, p l}^{2}\left(D, l_{0}\right)+\frac{0.51 \sigma_{1}^{2}\left(1+0.69 \sigma_{1}^{12 / 5}\right)^{-5 / 6}}{1+0.9 d^{2}+0.621 d^{2} \sigma_{1}^{12 / 5}}\right]-1,
$$

The Aperture Averaging factor is defined by, 


$$
A A F=\frac{\sigma_{I, p l}^{2}(D)}{\sigma_{I, p l}^{2}(0)} .
$$

\section{MATERIALS AND METHODS}

\subsection{Experimental Site}

The outdoor experimental setup has been completed and aligned ready for data collection at different weather conditions. One of the outdoor experimental locations is chosen to be the Odeon Parking Lot at Bilkent University's Main Campus in Ankara, Turkey. The Length of the beam path is about 250 meters. The declination of the beam path is approximately $3.42^{\circ}$. The height of the transmitters and receivers is 85 centimeters above the ground inducing strong turbulence to the propagating beam. The second setup is chosen as the Kim Engineering Building Parking Lot, University of Maryland at College Park, which represents a distance between the receiver and transmitter of about 120 meters. The selected site represents a uniform plain that is approximately 1.2 meters high above the ground to represent strong turbulence measurements. The convenient line of sight chosen, has a green bush and a tree barrier on one side so it is suitable for creating big eddies along the way. The site is also close to power plugs at both ends of our experiment and the ground level is homogeneous from start to end which all play an important role in choosing our experimental location. Figure 1 shows a satellite picture of the experimental locations chosen for the outdoor strong turbulence experiment.
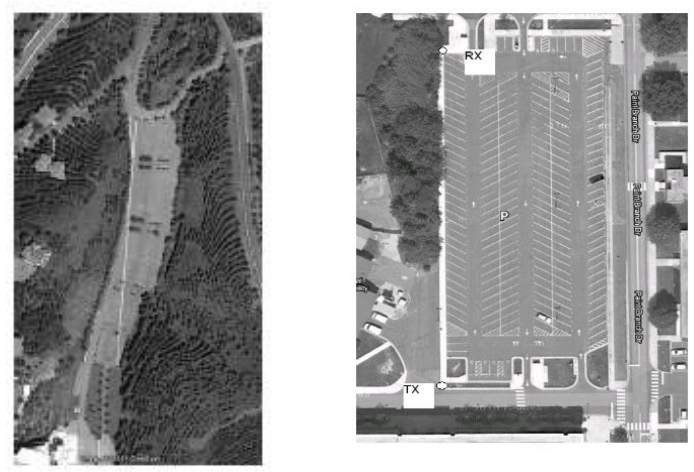

\subsection{Experimental Setup}

Fig. 1. Strong Turbulence Experimental Sites.

We have used a $35 \mathrm{~mW}$ He-Ne laser with a 30x beam expander with a directional freedom we got with the special heavy-duty tripod on the transmitter side. In the receiver side, we have collected the beam with a Celestron telescope and stored the turbulence induced beam shape with the help of our focus adaptor and a CCD camera. We have used a Pulnix camera model M1402-CL with 30fps and 1392 x 1040 monochromatic resolution settings.

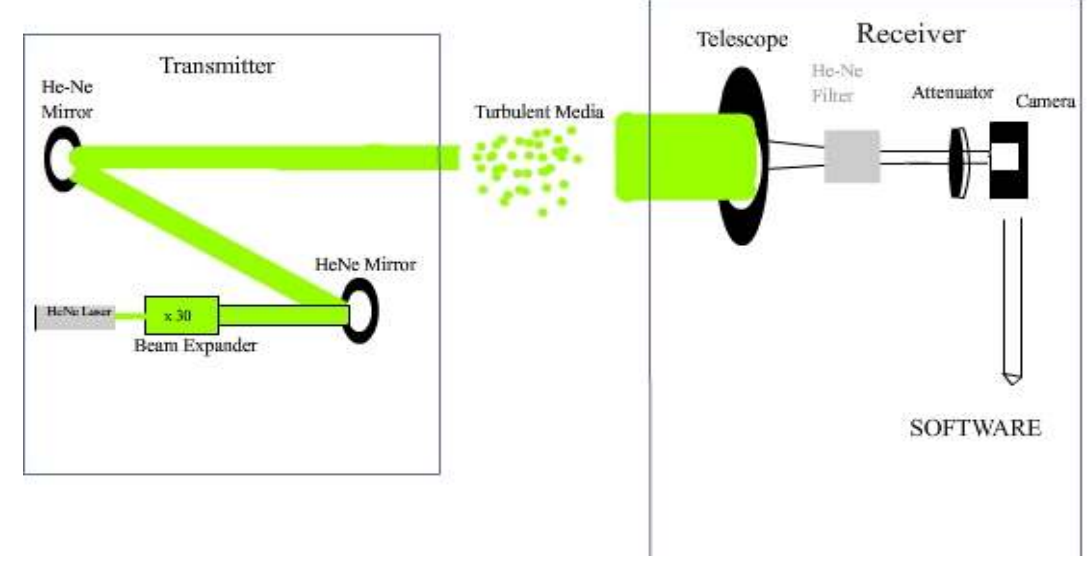

Fig.2. Overall Schematic of the experiment 
The general schematics can be seen in Figure 2. The transmitter part as shown in Figure 3 was setup on a portable breadboard with a rigid enclosure built specially to protect the equipment and optics from the rain and severe weather conditions. Since it is a flat, open area to be used for our outdoor link, we should be expecting strong winds and heavy rain for our setup. Our setup is strong and rigid enough to bear those winds and well enclosed for the water to leak. The enclosed transmitter setup is $15 \mathrm{~kg}$ excluding the heavy duty tripods. A rifle scope is placed at the top of the transmitter enclosure for the use in the alignment of the beam. The electric power is supplied from electric utility poles that exist in the experimental area.

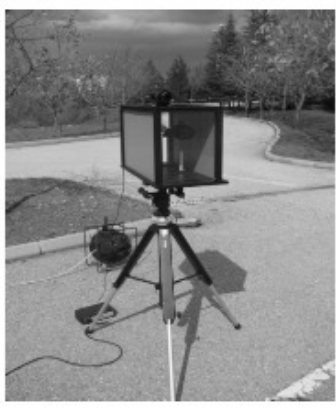

Fig. 3. Transmitter Side.

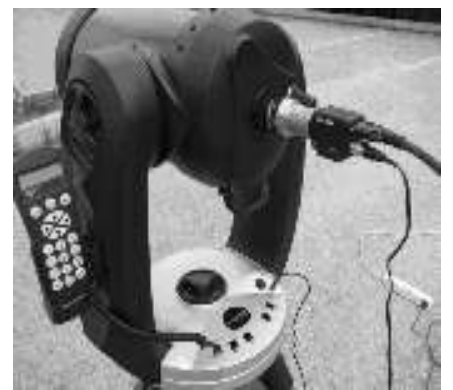

Fig. 4. Receiver.

The receiver side consists of the Schmidt-Cassegrain telescope followed by the He-Ne filter and the CCD camera. The images are taken by a CCD camera which is connected to the frame-grabber card onto a laptop for capturing frames into AVI files. The receiver part shown in Figure 4 is about $31.5 \mathrm{~kg}$ and is mounted on heavy duty tripods. An adapter has been especially constructed from aluminum for holding the He-Ne Filter to the telescope and the camera to the filter. With the help of the rifle scope, the alignment process has become an easy process. We used big white boards on the receiver side to help us center the beam onto the telescope. As the Gaussian beam travels over 250 meters, it expands in size and we center the beam at the receiver side to ensure the center part of the beam is seen by the telescope allowing us to use the plane-wave approximation in our calculations.

Weak turbulence experimental locations as well as the optical instruments for the outdoor turbulence experiment are mentioned in our previous works [2].

\subsection{Date Acquisition}

The saturation at the CCD camera was critical. Depending on the humidity of the weather, we had to change the attenuation level of the beam that goes through the camera. For us to have a data with most of the information that we are interested in, the dynamic range of the images' pixels must be well studied during the experiment and the attenuation should be chosen accordingly.

The method of choosing high attenuators and trying to increase the intensity of the image with the camera's built-in gain settings is observed to be unsuccessful. The intensity amplifier had just added a constant value through the doughnut shape and the turbulence effects are somehow averaged over spatial values and temporal values. A moderate turbulence induced sequential images with high gain settings can be seen in Figure 5 .

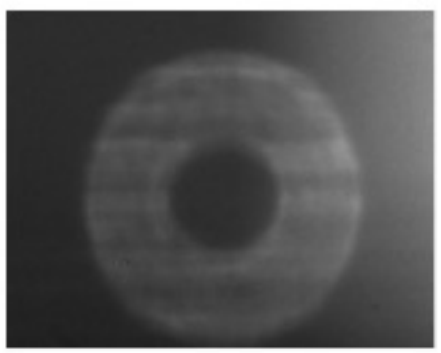

Fig.5. Faulty Image Settings. 
Before starting on storing the experimental data, images are taken as a sample and image processing techniques are applied in order to find where exactly the beam lies. Then, the histogram of the images is taken in order to compare the contrast of them. Low gain settings are applied in order to get rid of the amplifier noise in the image and the attenuator that results with the most dynamic contrast is chosen.

As we can see from Figure 6, the faulty image has interference with the background. The separation between the information and the noise seems to be interfering with each other. On the other hand when we look at the histogram of the appropriate image in Figure 7, the separation is much clearer. The transition from the noise (background) to information is almost flat. Once proper attenuation settings are chosen and the appropriate images are located, image processing techniques using frame analysis are applied to find the aperture averaging factor. Such processing techniques applied to the images will be discussed in detail in this paper. A sample strong turbulence induced image can be seen in Figure 8.

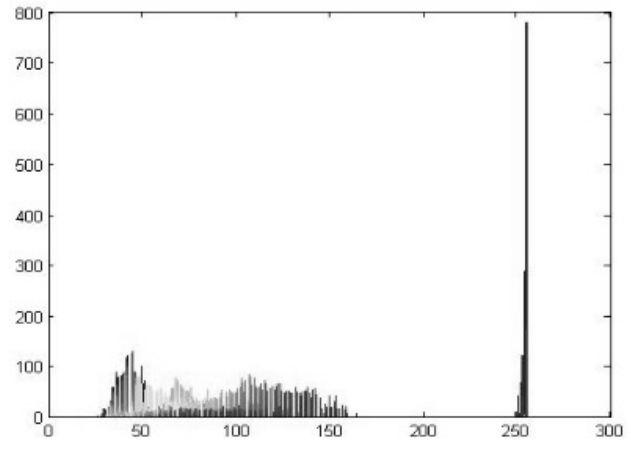

Fig.6. Histogram of faulty image settings.

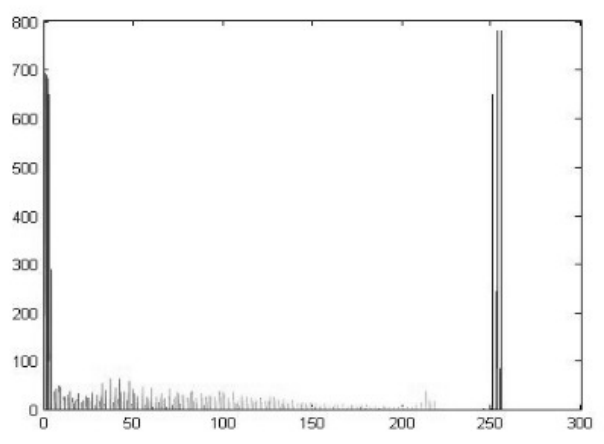

Fig.7. Histogram of right image settings.

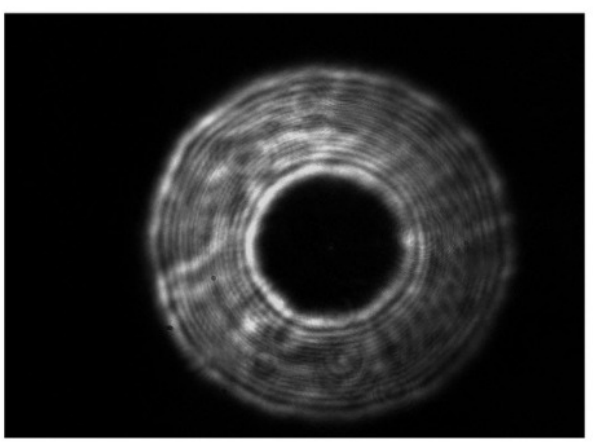

Fig.8. Intensity Image with right settings.

\subsection{Technique on masking the image}

The ideal image at the receiver side without any turbulence should be seen as a perfect donut shape. In terms of intensity distribution, we can safely approximate the same intensity value at each point (plane wave approximation) since $D<<\sqrt{\lambda L}$. Existing data from the University of Maryland setup have been used to test our software. The dark area occurs in the middle because of the small diameter mirror of the Schmidt-Cassegrain telescope at the receiver side blocking the central portion of the image. 


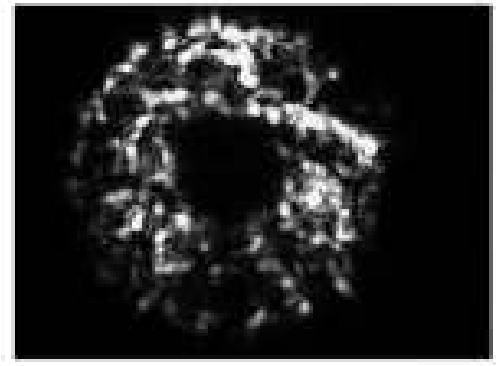

Fig.9. Turbulence induced image.

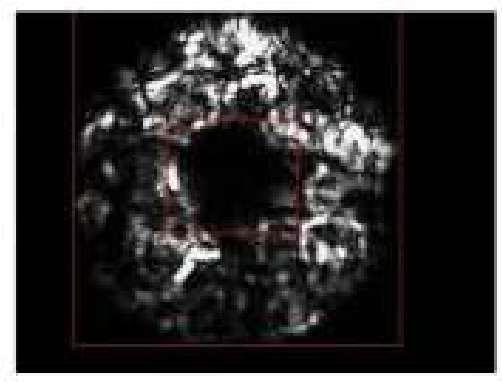

Fig.10. Region result of the software.

Due to turbulence induced fluctuations, the intensity distribution of the image at the receiver becomes rather plain and simple as shown in Figure 9.The result of the region software can be seen in Figure 10. In order to analyze the image we have to set some deterministic boundaries for where our information lies. Mainly we can determine these boundaries by only two parameters, radius of the inner circle and radius of the outer circle. The preceding method will explain the steps of succession in our software.

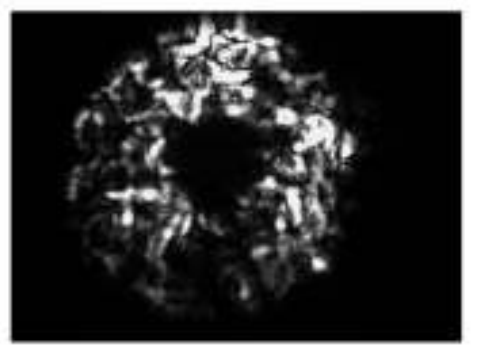

Fig.11. Turbulence induced problematic image.

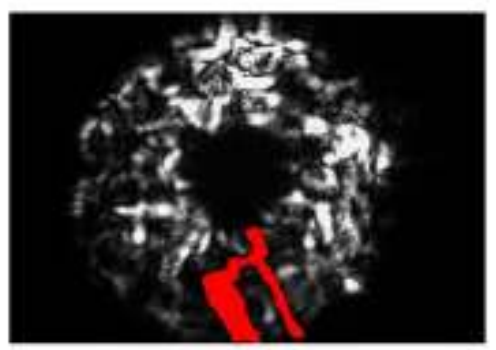

Fig.12. Region that is causing problems.

At first, we subtract the background noise, which is only one frame that is taken from the camera when the laser is off, caused by the noise that is coming from thermal radiations or sun rays. After that process, a smoothing operation is needed to reduce the effects of the turbulence generated paths from the inner circle to the outer circle. Examples of such images can be seen in Figure 11 and Figure 12.

Then we convert a binary image from the result to see where our information lies. Smoothing operation gives us a better understanding of the inner and outer boundaries of the image. The difference caused by the smoothing operation can be clearly seen in Figures 13 and 14.

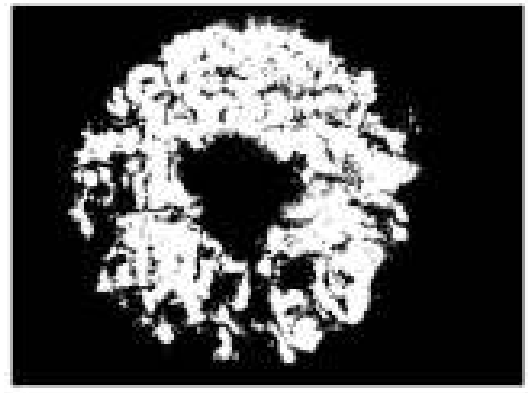

Fig.13. Binary image without smoothing

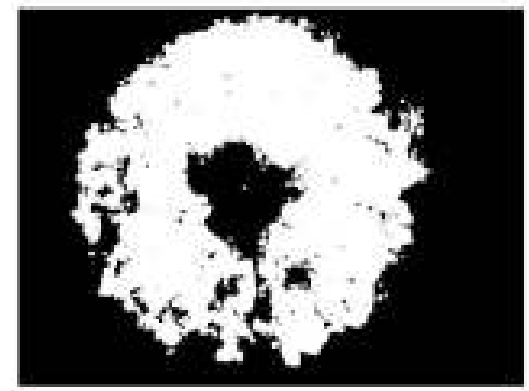

Fig.14. Binary image after smoothing

Labeling each of the closed boundaries gives us several regions inside our image matrix. The biggest one of these should be able to give us the outer radius of the image. For the region with the biggest area, we are able to fit a square or a circle after that by calculating the minimum and maximum location points in the $\mathrm{x}$-axis and the y-axis. 
The same operation applies for the inner circle. The only difference is that the region of the inner circle is assumed to be higher than $1 / 8$ of the size of the image considering the structure of Schmidt-Cassegrain telescope.

Once the inner and outer radii of the captured frames are determined, the same framing technique discussed previously is used in calculating the aperture averaging factor $[2,3]$. The better masking technique discussed above produced a more efficient aperture averaging factor results.

The energy in the atmosphere under the effect of the inertia break up into eddies and form a continuous pack of inner group starting from $l_{0}$ (inner scale of turbulence) to $L_{0}$ (outer scale of turbulence) with either velocity fluctuations or temperature fluctuations or both. The scale sizes that are smaller than inner scale $l_{0}$, are assumed to be dissipated in the air as heat.

In terms of velocity fluctuations in the air which is appearing as wind, inner scale is defined by $l_{0}=\left(v^{3} / \varepsilon\right)^{1 / 4}$ where $\varepsilon$ is the energy dissipation rate and $v$ is the viscosity. Outer scale, $L_{0}$ is proportional with the $\varepsilon^{1 / 2}$; and generally assumed to be proportional with strength of the change in the path averaged mean flow velocity [10].

The general ideas of the velocity fluctuations also have been applied to the temperature fluctuations $\varphi=T+\alpha_{a} h$ where $\varphi$ is the potential temperature, $\alpha_{a}$ is the adiabatic rate of decrease in the temperature and $h$ is the height above the ground. So the inner scale is defined by $l_{0}=5.8\left(D^{3} / \varepsilon\right)^{1 / 4}$ where $D$ is the diffusivity of heat in the air $\left(m^{2} / s\right)[11]$.

Near to the ground, inner scale of turbulence is typically between $1 \mathrm{~mm}$ to $10 \mathrm{~mm}$ [10]. So the study of inner scale effects without a scintillometer is somewhat hard. In the work of Andrews et al.[11], inner scale have been measured between 1 to $10 \mathrm{~mm}$ in strong turbulence regimes. In this work with scintillation index calculations, inner scale factor is assumed to be $5 \mathrm{~mm}$. The effect of the inner scale factor can be seen in Figure 15. The error factors in strong turbulence analyses can very well be a cause of the effect of the difference of the real inner scale and the assumed. It is very clear from Figure 15 that even though there isn't much of a difference when the induced turbulence is weak, as the Rytov variance gets bigger, the effect of the inner scale becomes vital.

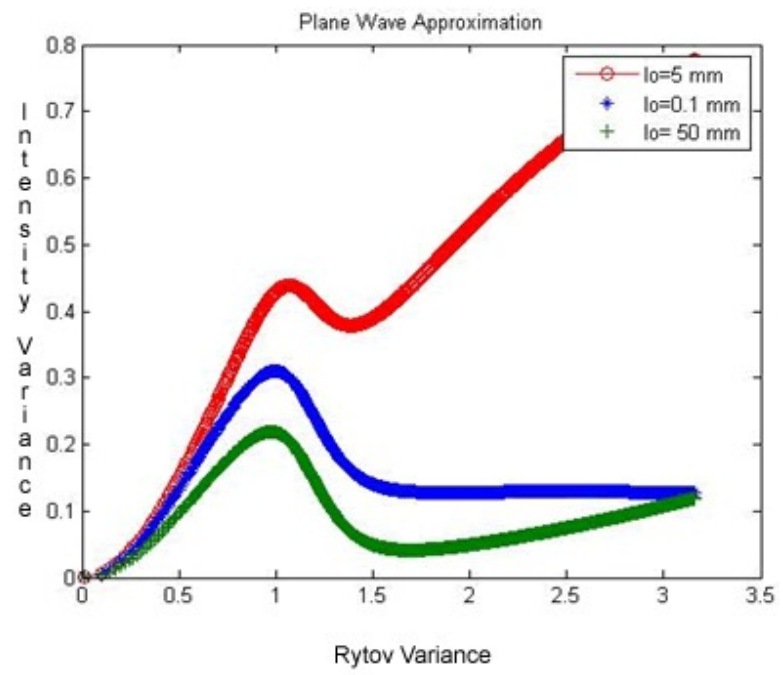

Figure 15. Intensity Variance vs. Rytov Variance for varying inner scale factors. 
Effect of the receiving aperture is clearly seen in Figure 16. With the right amount of receiver size it is clear that even though the Rytov variance gets bigger, the intensity variance can be optimized for optical communication regardless of the inner scale value.

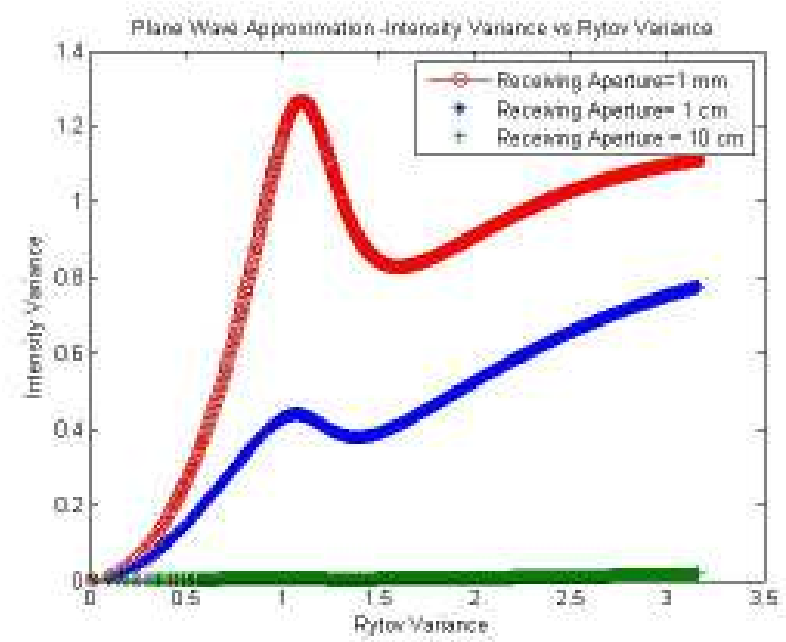

Figure 16: Intensity Variance vs. Rytov Variance for varying receiving aperture sizes.

\section{RESULTS}

Variations of turbulence strength estimation methods as well as frame image analysis techniques are then been applied to the experimental data in order to study the effects of different parameters on the result.

Aperture averaging factor for different centers on the doughnut shape of the image can be seen in Figure 17. In some of the center points that are chosen, we weren't able to observe the expected decline on the Aperture Averaging (AA) curve for limited amount of frames. This phenomena starts to become a problem when the turbulence level starts to gets stronger. The reason for that is the sensing time of the photo detector starts to become not enough and the amount of frame that is need for calculating the AA increases. In order to get rid of these false advertising and decreasing the computational complexity, we have used PAAA (Point Averaged Aperture Averaging). Different centers on the receiving beam have been chosen and for each of the equal-distance center points on the receiver beam the AA factor for varying sizes of the receiver has been calculated.

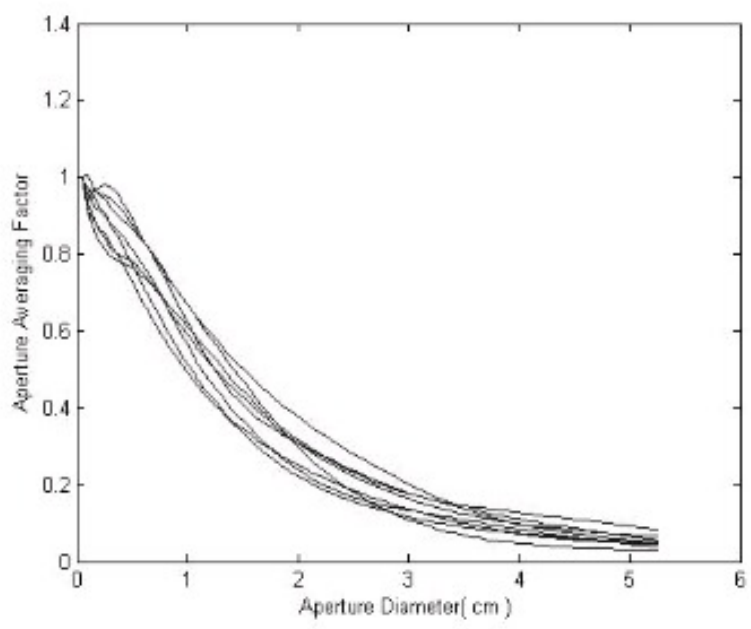

Fig.17. Aperture averaging factor for different center points on the beam, $C_{n}{ }^{2}=3.24 \times 10^{-14}$. 
It is found that the resulted AA curve can be reached by applying PAAA with much less frame number. Ratio of the number of frames that has been used in our study is " 8 " with $\sim 1 \mathrm{~ms}$ shutter speed. Future study can be made by investigating the effects of number of center points that has been used and the shutter speed. The final characteristic PAAA factor that we have for strong turbulence $\left(\mathrm{C}_{\mathrm{n}}{ }^{2}=3.24 \times 10^{-14}\right)$ data can be seen in Figure 18 .

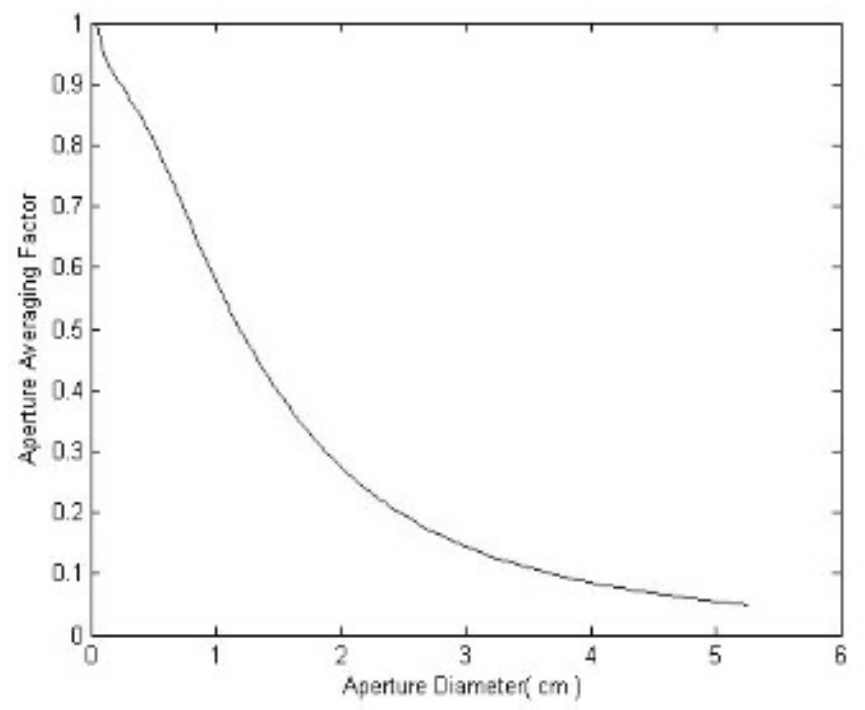

Fig.18. Point Averaged Aperture Averaging Factor for strong turbulence Data, $C_{n}^{2}=3.24 \times 10^{-14}$.

In Figure 19, we are comparing the PAAA factor for our two strong turbulence experiment results. For this Experiment only the distance between the transmitter and the receiver are arranged to be 120 meters for both of the Experimental Sites. The height of the experiment is clearly a big factor in strong turbulence measurements. We have about $35 \mathrm{~cm}$ differences in terms of height above the ground between the Bilkent University and the UMD setup which create a big difference in the declination of the AA curves. $C_{n}^{2}$ values are measured to be $5.21 \times 10^{-13}$ in the Bilkent University Setup and $3.24 \times 10^{-14}$ in the UMD Setup.

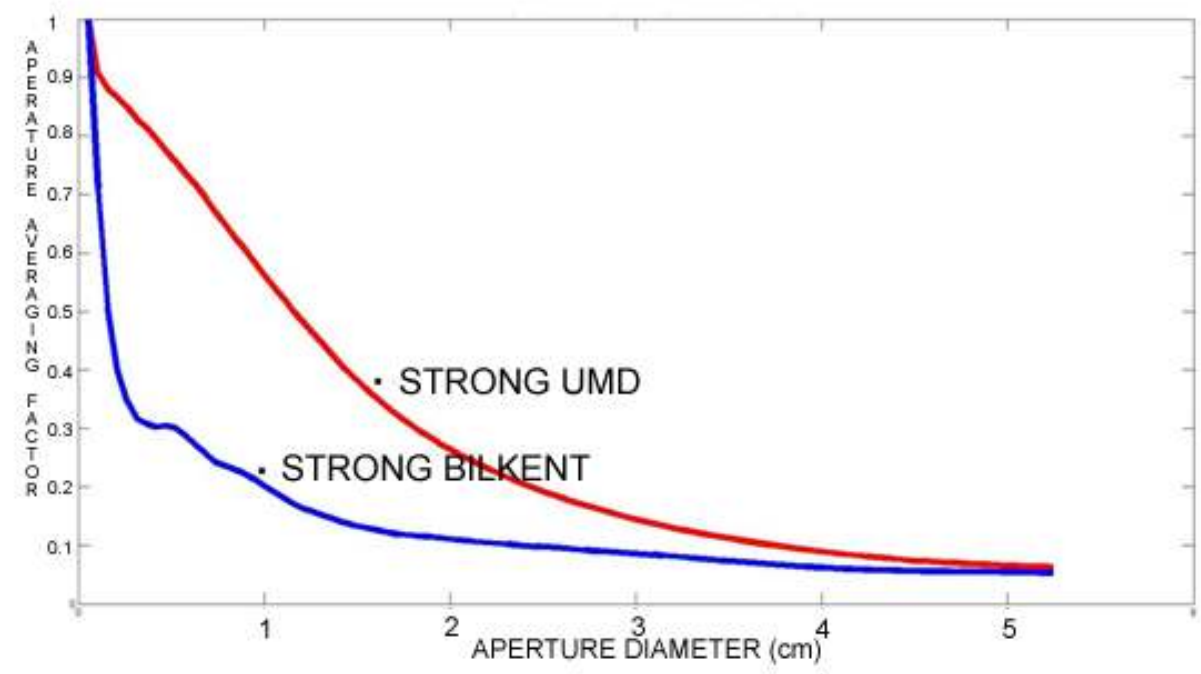

Fig.19. AA Factors for two strong turbulence Setups, Bilkent $C_{n}^{2}=5.21 \times 10^{-13}, \mathrm{UMD}_{\mathrm{n}}{ }^{2}=3.24 \times 10^{-14}$. 
It is seen from Figure 19 that we are getting stronger turbulence data from the Bilkent University experiment with a declination of the AA curve being the steepest. Even though the air conditions were more suitable for creating stronger turbulence in the UMD setup, the closeness to the ground allowed us to have stronger turbulence induced beam at the receiver side.

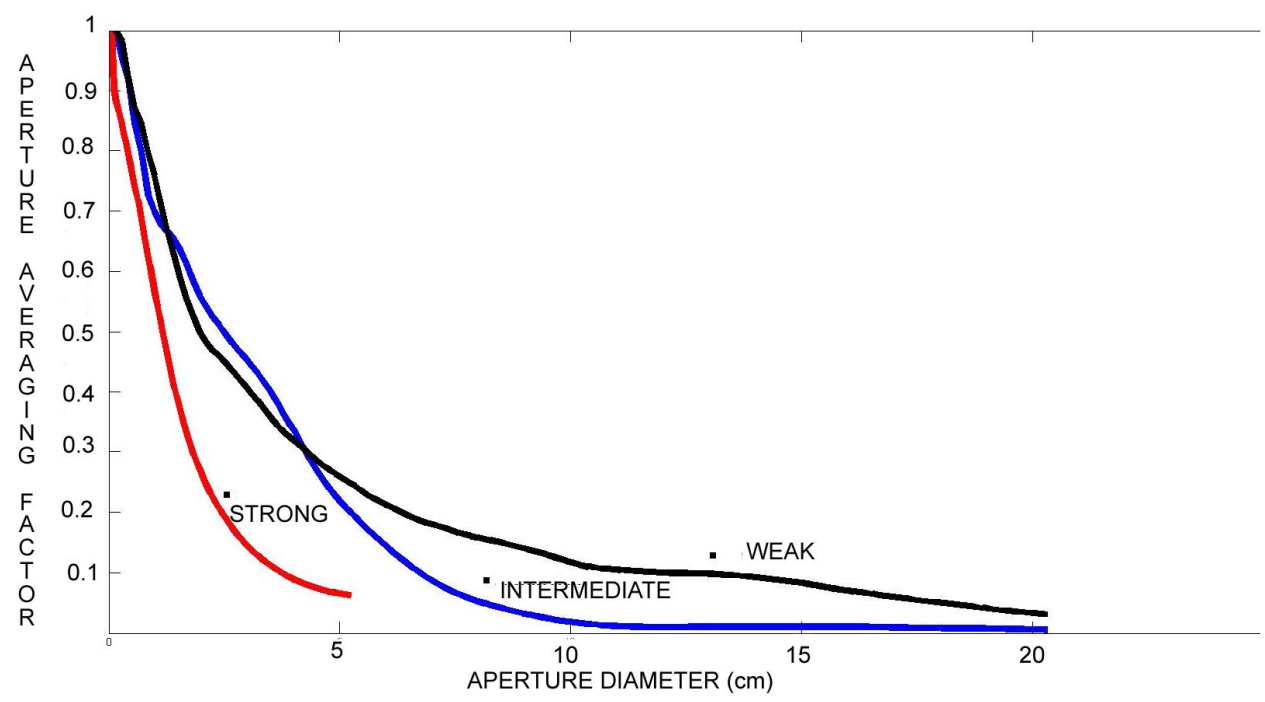

Fig.20. Combined AA curves for different turbulence regimes, Strong $C_{n}{ }^{2}=3.24 \times 10^{-14}$, Intermediate $\mathrm{C}_{\mathrm{n}}{ }^{2}=2.57 \times 10^{-15}$ Weak $\mathrm{C}_{\mathrm{n}}{ }^{2}=8.63 \times 10^{-16}$.

Figure 20 shows Aperture Averaging curves for different turbulence regimes. We can conclude from that figure that the stronger the turbulence level, the steeper the initial decline in the AA Factor.

Theoretical results have been calculated for each central pixel point of the received beam. The general error margin is calculated by

$$
\text { Error }=\frac{1}{\max i} \sum_{i=1}^{\max i} E R R_{i}
$$

where

$$
E R R_{i}=100 x \frac{\mid \text { Theo }_{i}-\operatorname{Exp}_{i} \mid}{\operatorname{Exp}_{i}}
$$

In Figures 21-23, we compare the experimental results of the aperture averaging factors with the Scintillation index model for varying $C_{n}^{2}$ values, path lengths $=120 \mathrm{~m}$ and $951 \mathrm{~m}$ and a wavelength $=633 \times 10^{-9} \mathrm{~m}$. 


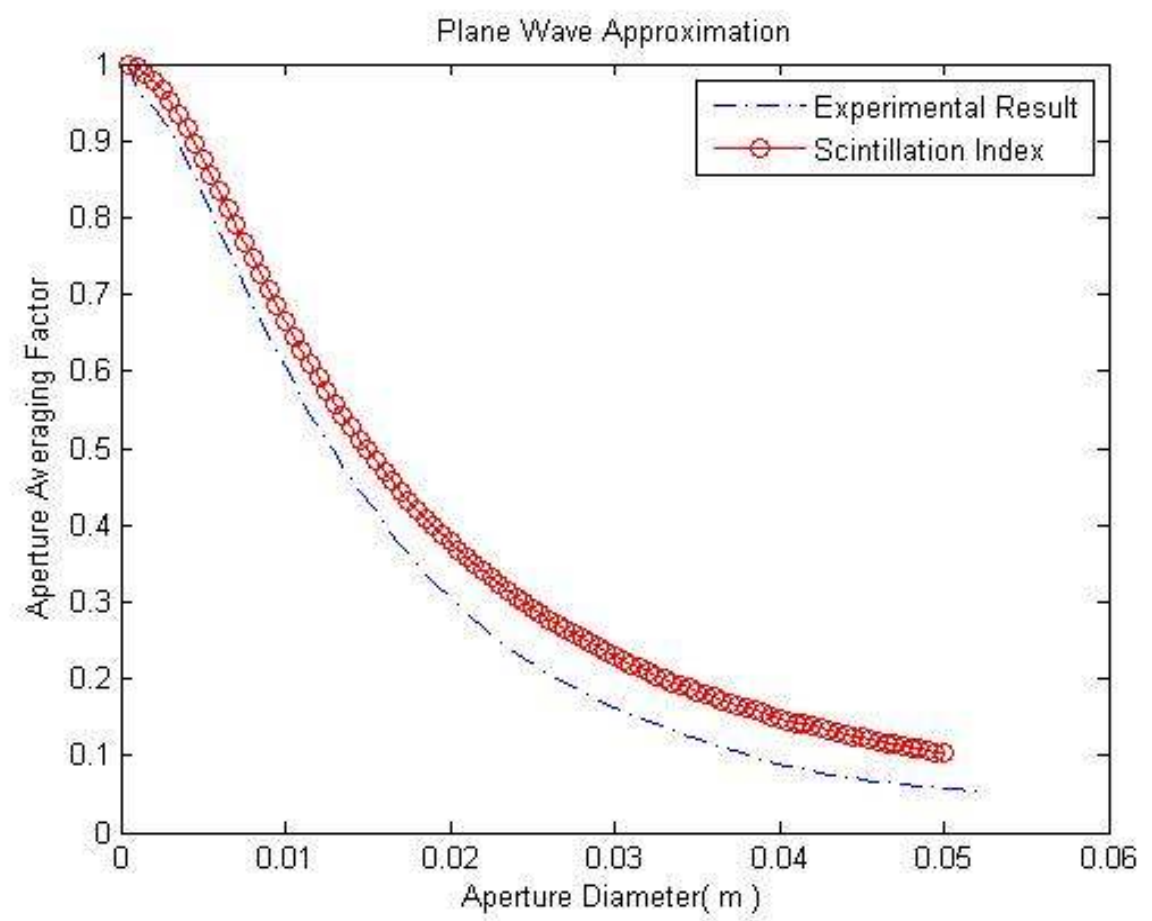

Fig.21. Experimental Results and Theoretical Results of Aperture Averaging Factor for $\mathrm{C}_{\mathrm{n}}{ }^{2}$ structure parameter of $3.24 \times 10^{-14}$, Resulted error margin is $22.97 \%$.

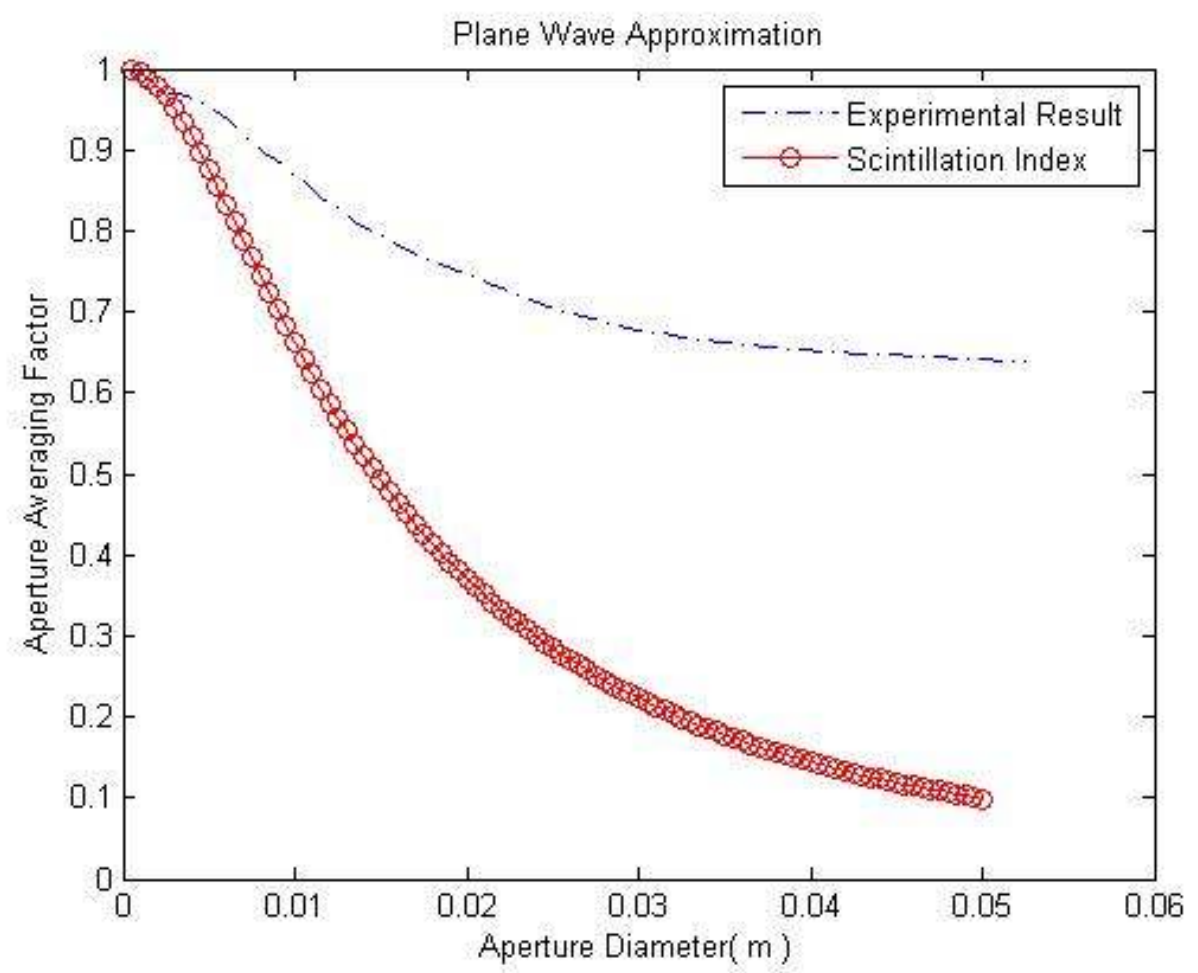

Fig.22. Experimental Results and Theoretical Results of Aperture Averaging Factor for $\mathrm{C}_{\mathrm{n}}^{2}$ of $9.62 \times 10^{-13}$, Resulted error margin is $50.69 \%$. 


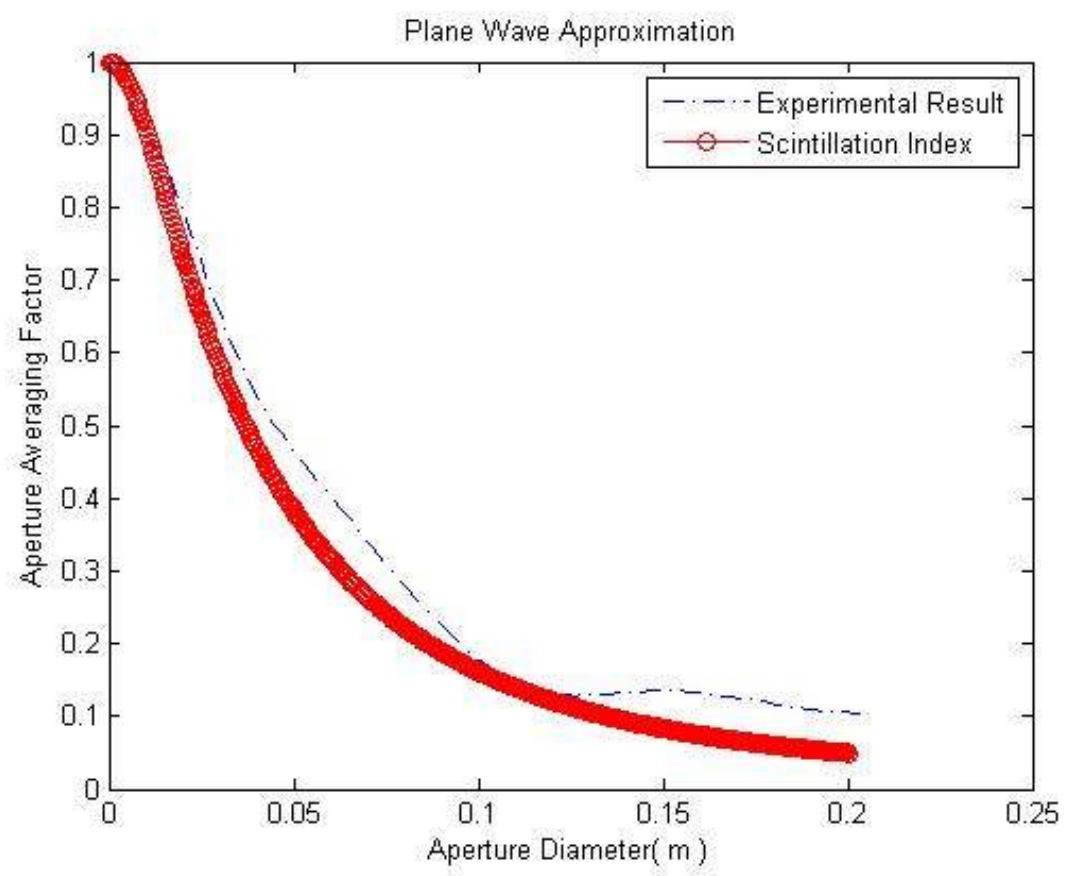

Fig.23. Experimental Results and Theoretical Results of Aperture Averaging Factor for $\mathrm{C}_{\mathrm{n}}{ }^{2}$ of $1.19 \times 10^{-15}$, link length of $951 \mathrm{~m}$, and the Resulted Error margin is $12.72 \%$.

As it can be seen from the analysis of the strongest segment of data in Figure 22, at a $5 \mathrm{~cm}$ aperture diameter there is a huge error on the convergence of the Aperture Averaging curve with the theoretical data. According to our findings, as the $\mathrm{C}_{\mathrm{n}}{ }^{2}$ increases, prediction of the theoretical model stays below the experimental resulted curve but as $\mathrm{C}_{\mathrm{n}}{ }^{2}$ decreases the resulted theoretical curve stays above the experimental result. For relatively weak turbulent regimes, the theoretical model gives us a pretty good match with the experimental data as can be seen in Figure 23. However we still should not be convinced with the results since for an accurate Aperture Averaging Analysis the most important part is the Aperture Averaging factor for the biggest diameter and we don't see the converged value of the model matching with the experimental findings.

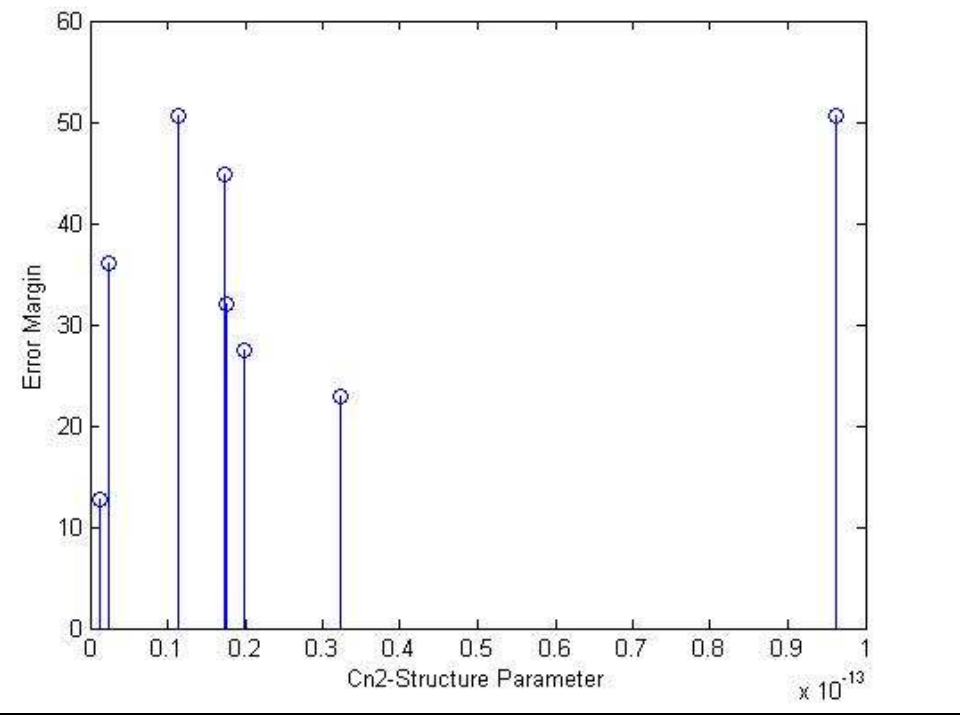

Fig.24. Error Margin for $5 \mathrm{~cm}$ diameter receiver vs. the $\mathrm{C}_{\mathrm{n}}^{2}$ structure parameter. 
We can have a look at the overall picture modeling of the atmospheric turbulence in Figure 24. It is apparent that we have some downsides when it comes to strong turbulence regimes. As the AA Factor curve converges, scintillation index model is working fine with weak-to-moderate turbulent regimes but when the $\mathrm{C}_{\mathrm{n}}{ }^{2}$ of the turbulent induced beam gets bigger the theoretical results stays very low when you compare with the experimental results which makes the model useless practically as it can be seen in Figure 22.

\section{CONCLUSIONS}

In this study, a strong turbulence experimental setup for investigating the effects of AA Factor in FSO communication system is proposed. PAAA is proposed for analysis of AA Factors in strong turbulence regimes with less computational complexity. With the help of our strong turbulence experimental setup we have tested the Scintillation Index model which is the most accepted model in literature. It has been found that there is a good correlation between the Scintillation Index model and our experimental data for the AA factor when it comes to weak-to-intermediate turbulence regimes. However, for the strong turbulence regime, the model seems not to match the AA factor results from our experimental turbulence data. In addition, the aperture averaging factor for differenct turbulence regimes have been investigated. It was shown that the stronger the turbulence, the steeper the initial decline in the Aperture Averaging curves. The effect of aperture size at the receiver and the inner structure parameter in theory were also analyzed to monitor the change in the intensity variance as the Rytov variance increases. It has been seen that in theory using the scintillation index model for strong turbulence regimes

$\left(\sigma_{R}^{2}>>1\right)$, the inner structure parameter is commanding on the value of the intensity variance at small diameter values.

\section{ACKNOWLEDGEMENTS}

We would like to acknowledge the support of Bogazici University Research Foundation (BAP Project Numbers: 07A204D and 07S102) and TUBITAK (Project Number: 108E205).

\section{REFERENCES}

[1] Davis, C. and Smolyaninov, I. "The effect of atmospheric turbulence on bit-error-rate in an on-off-keyed optical wireless system," Free-Space Laser Communication antd Laser Imaging, Proc. SPIE., vol. 4489, 126-137, (2002).

[2] Yuksel, H. "Studies of the effects of atmospheric turbulence on free space optical communications," Ph.D. Thesis, University of Maryland, (2005).

[3] Yuksel, H., Milner, S. and Davis, C. "Aperture averaging for optimizing receiver design and system performance on freespace optical communication links,” J. Opt. Netw. 4, 462-475 (2005).

[4] Andrews, L. C. [Laser Beam Propagation Through Random Media. Bellingham], SPIE Optical Engineering Press, Washington, (1998).

[5] Andrews, L. C., [Laser Beam Scintillation with Applications], SPIE Press, Bellingham, Washington, (2001).

[6] Andrews, L.C., Philips, R.L., Hopen, C.Y. and Al-Habash, M.A. “Theory of Optical scintillation" J. Opt. Soc. Am., Vol. 16, No. 6, (1999).

[7] Dunphy, J. and Kerr, J. "Scintillation measurements for large integrated-path turbulence" J. Opt. Soc. Am., Vol. 63, No. 8, (1973).

[8] Andrews, L. C., Phillips, R. L. and Hopen, C. Y. "Aperture averaging of optical scintillations: power fluctuations and the temporal spectrum," Wave Random Media Vol. 10, (2000), p. 53-70.

[9] Andrews, L. C., Phillips, R. L. and Hopen, C. Y., [Laser Beam Scintillation with Applications], SPIE Optical Engineering Press, Bellingham, Washington, (2001).

[10] Wasiczko, L. "Techniques to Mitigate the Effects of Atmospheric Turbulence on Free Space Optical Communication Links," Ph.D. Thesis, University of Maryland, (2004).

[11] Andrews, L. J. "Field Guide to Atmospheric Optics" SPIE Field Guides, Vol. FG02,SPIE Press, Bellington, Washington. 